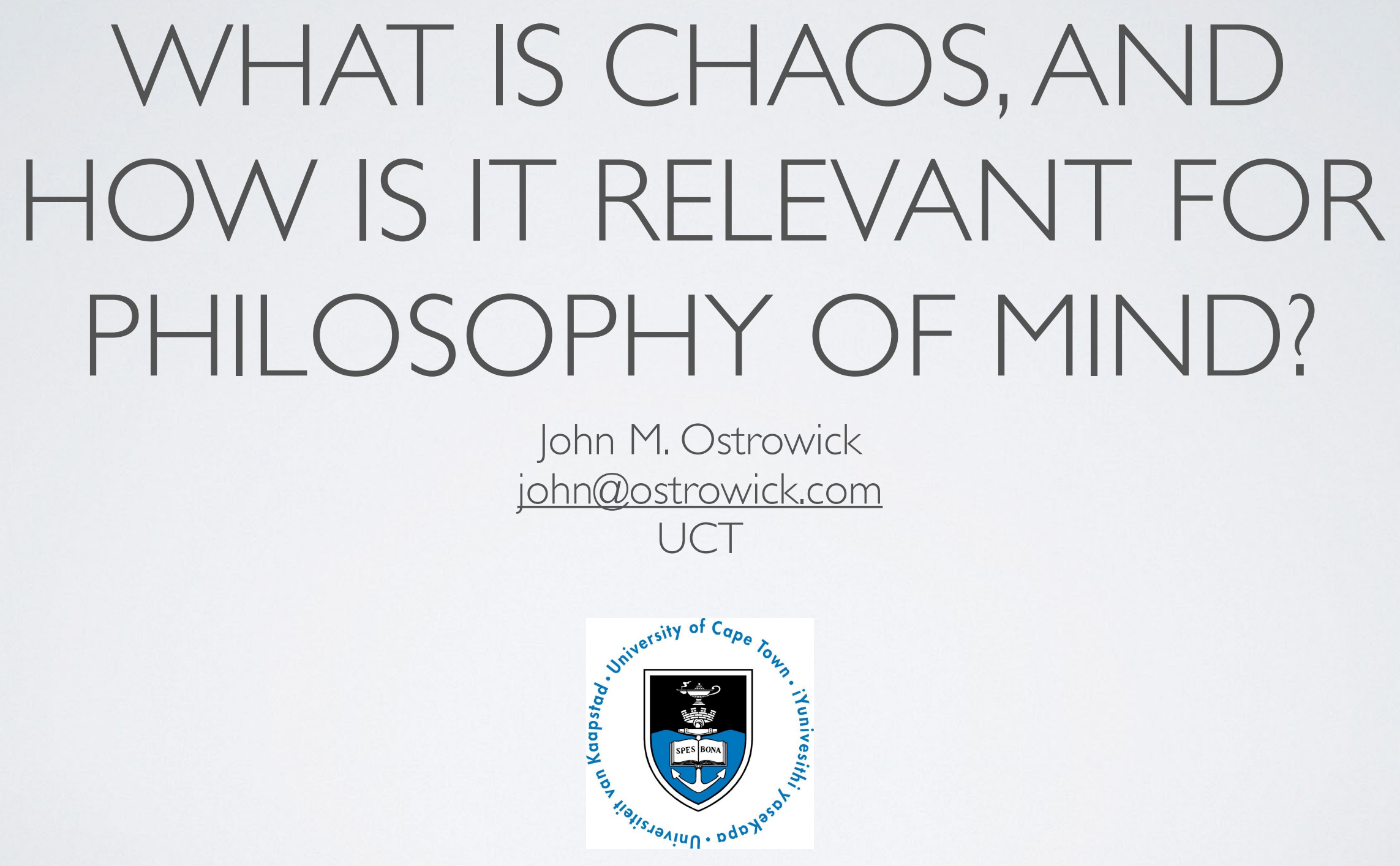




\section{ABSTRACT}

This paper argues, in agreement with Neuringer, Beggs and others, that if we wish to scientifically characterise human choice we have to work with a probabilistic or chaos-derived model. This has a number of implications for philosophy of mind. Firstly, it is in theory possible to describe human behaviour with some form of law-like equation; it's just a matter of figuring out what equation best captures the states of our central nervous system. Secondly, it means that our choices are not random but are chaotic: deterministic, but just hard to predict due to internal complexity. According to the current research, our actions are most likely caused by 'precipitated avalanches' of neural activity, which are difficult to predict due to chaos. 


\section{INTRODUCTION}

- Chaos Mathematics is the study of dynamic systems and the equations that represent them.

- There are three types of randomness:

- Entropy (Second Law of Thermodynamics): the amount of additional information needed to specify the exact physical state of a system; a statistical measure of randomness, regardless of how that randomness came about.

- True, Quantum or Stochastic randomness is the type of disordered state generated by quantum fluctuations. Truly random.

- Chaos: pseudo-randomness; seems to be random but in fact deterministic. Extremely sensitive to small changes and initial conditions. Divergence happens rapidly, and in the longer term, results in unpredictability and irretrievability (as per the Second Law of Thermodynamics). Locally unpredictable, globally stable. 


\section{INTRODUCTION}

- Non-linear, we would find that it is impossible to extrapolate from the graph of known data points, to where the data will go next (*). Consider the Ideal Gas Law, $P V=n R T$. Not sensitive to minor errors. Chaotic equations differ in those two respects; they're neither linear, nor are they tolerant of minor errors.

- What chaos attempts to do is explain why e.g. scatterplot points do not sit on a neat line. Real-world data isn't neat or ideal.

- Examples include pseudo-random numbers generated by a computer algorithm, the structure of organisms, the weather, turbulence and swirls in smoke and fluids generally, the pattern of veins in a leaf, snowflake structures, the evolution of organisms, population changes, market prices, and the path of a fork of lightning. Chaos may be the rule and linear dynamics the exception. 


\section{EXAMPLES}
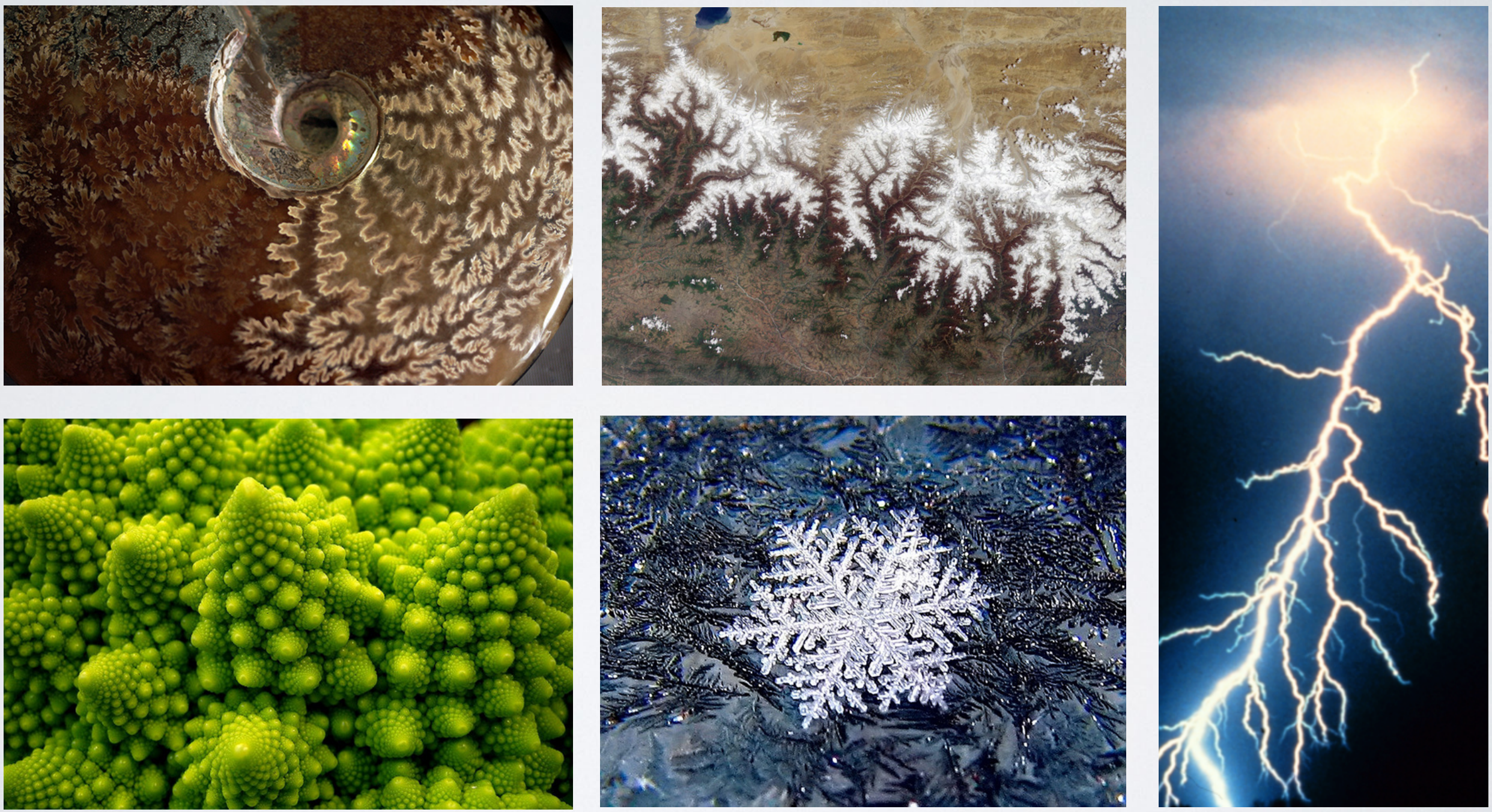

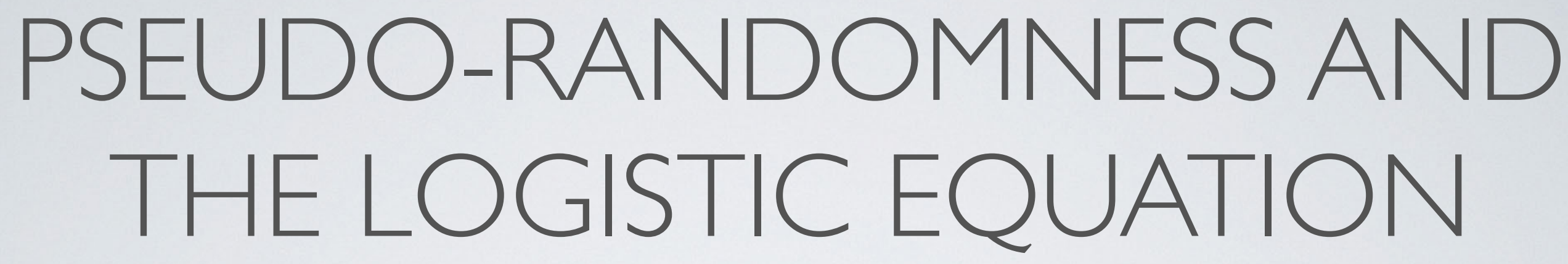

- Computers do not generate truly random numbers. On a computer, to generate a 'random' number, one typically provides a "seed number," which is then passed through an algorithm to render it unrecognisable, and then one then restricts it to a desired range of numbers. Once the result is obtained, it is memorised as the seed number for the next iteration.

- The Logistic Equation:

$$
x_{\mathrm{n}+1}=r x_{\mathrm{n}}\left(1-x_{\mathrm{n}}\right)
$$

The range (or "period") $r=3.55$ to $r=4.0$ generates apparently random decimal numbers:

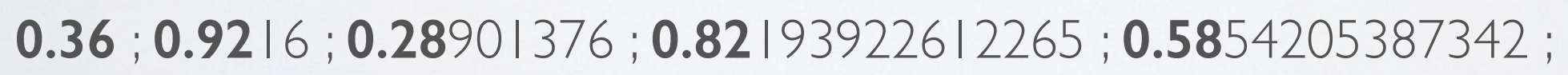

$0.9708 \mid 332624944 ; 0.11333924730376$ 


\section{FRACTALS AND ATTRACTORS}

- Chaotic equations are also marked by self-similarity or repetition, and as we mentioned, non-linearity. This self-similarity when graphed is known as a "fractal" or "attractor". (*)
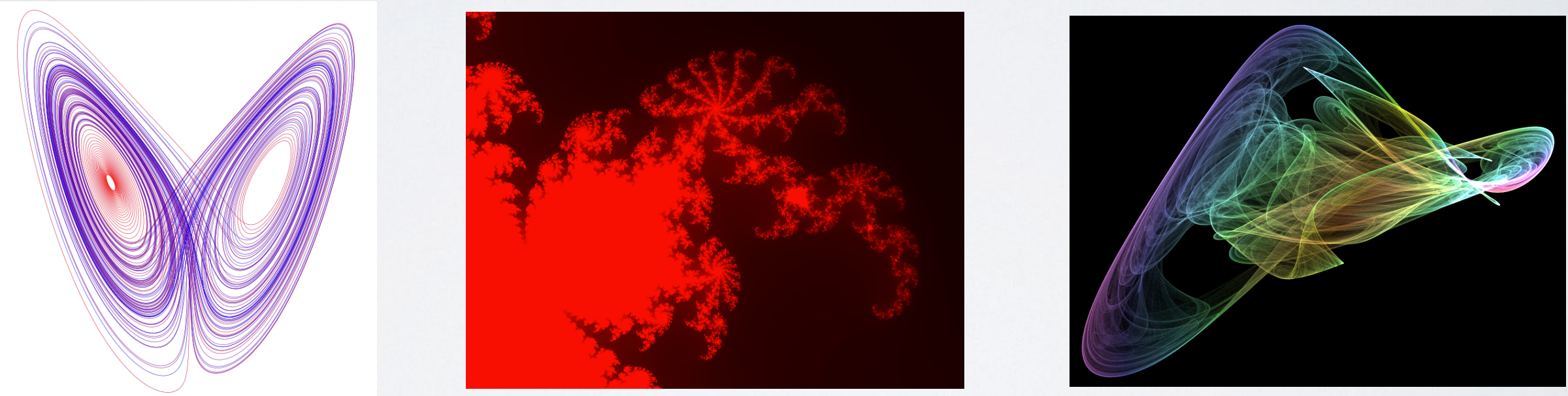


\section{CHAOSTHEORY AND PHILOSOPHY: RELEVANCE}

- Predictability and explanation in epistemology. One can no longer assume that there are straightforward deductively regular relationships between causes and effects. E.g. closure under entailment in Van Inwagen.

- It is unclear whether quantum states are relevant at the neurological level. We have significant tendencies of behaviour, which do appear in chaotic data, but do not appear in true stochastic data. Hence, perhaps chaos offers us some hope of understanding human volition. 


\section{ISTHE BRAIN CHAOTIC?}

- The brain is a complex system. Complex systems produce behaviours or results that are "greater than the sum of their parts," a phenomenon known as "emergence". Traffic is the easiest example of this. You cannot understand what traffic is by looking at a car's parts, or a car.Traffic is a function of how the car interacts with other cars, traffic laws, weather conditions, road conditions, other drivers, etc.

- The same applies to the brain. Looking at the neurons will not tell you what consciousness is like, or what decision would be taken. 


\section{IS THE BRAIN CHAOTIC?}

66

Have you ever experienced that eerie feeling of a thought popping into your head as if from nowhere, with no clue as to why you had that particular idea at that particular time? ... In reality, your brain operates on the edge of chaos. Though much of the time it runs in an orderly and stable way, every now and again it suddenly and unpredictably lurches into a blizzard of noise... The quintessential example of self-organised criticality is a growing sand pile. As grains build up, the pile grows in a predictable way until, suddenly and without warning, it hits a critical point and collapses. These 'sand avalanches' occur spontaneously and are almost impossible to predict... periods of stability [are] followed by catastrophic periods of instability that rearrange the system into a new, temporarily stable state." (Robson, 2009, pl) 


\section{IS THE BRAIN CHAOTIC?}

- Van Inwagen (defending incompatibilism, himself a libertarian), says in his "Consequence Argument" that if determinism is true, where $P$ is a future action choice, no-one would have a choice that $P$ if $P$ is caused by antecedent states $P_{0}$ and the laws of the universe L. However, given Chaos Theory, it seems very unlikely that the premises and mode of necessity in Van Inwagen's original argument could be correct, since Chaos Theory precludes complex systems such as $P_{0}$ and $L$ from necessitating only one particular subsequent state, such as $P$. 


\section{IS THE BRAIN CHAOTIC? NEURINGER ANDVOSS}

- Neuringer and Voss ran three experiments with the same human subjects. These subjects were asked to choose random numbers to predict what the computer would choose. The computer was outputting values from the Logistic Difference Equation (a variant on the Logistic Equation). The subjects' choices of numbers fell closely on a parabolic curve shown in the figure that follows. This shows that the choices were self-similar and algorithmic, even though they appeared, or were believed by the subjects, to be random. Neuringer et al. concluded that human choice is chaotic, and specifically follows the Logistic Difference Equation. 


\section{IS THE BRAIN CHAOTIC? NEURINGER ANDVOSS}

- By performing what is known as an autocorrelation, we can find correlations in a signal with itself, and therefore detect if it is stochastic, algorithmic or chaotic. A signal (series of numbers) which is self-similar is likely a chaos signal. If a signal looks random and does not autocorrelate, it is stochastic. A graph can be produced for any signal that has an ordered structure. In the case of the Logistic Equation, a parabola is produced (Fig below).

- (*) Lag-I autocorrelation is when $\mathrm{n}-\mathrm{I}: \mathrm{n}$. 


\section{ISTHE BRAIN CHAOTIC? NEURINGER ANDVOSS}

- Lag-I and Lag|,2 autocorrelations found by N\&V in their experiment.

(a)

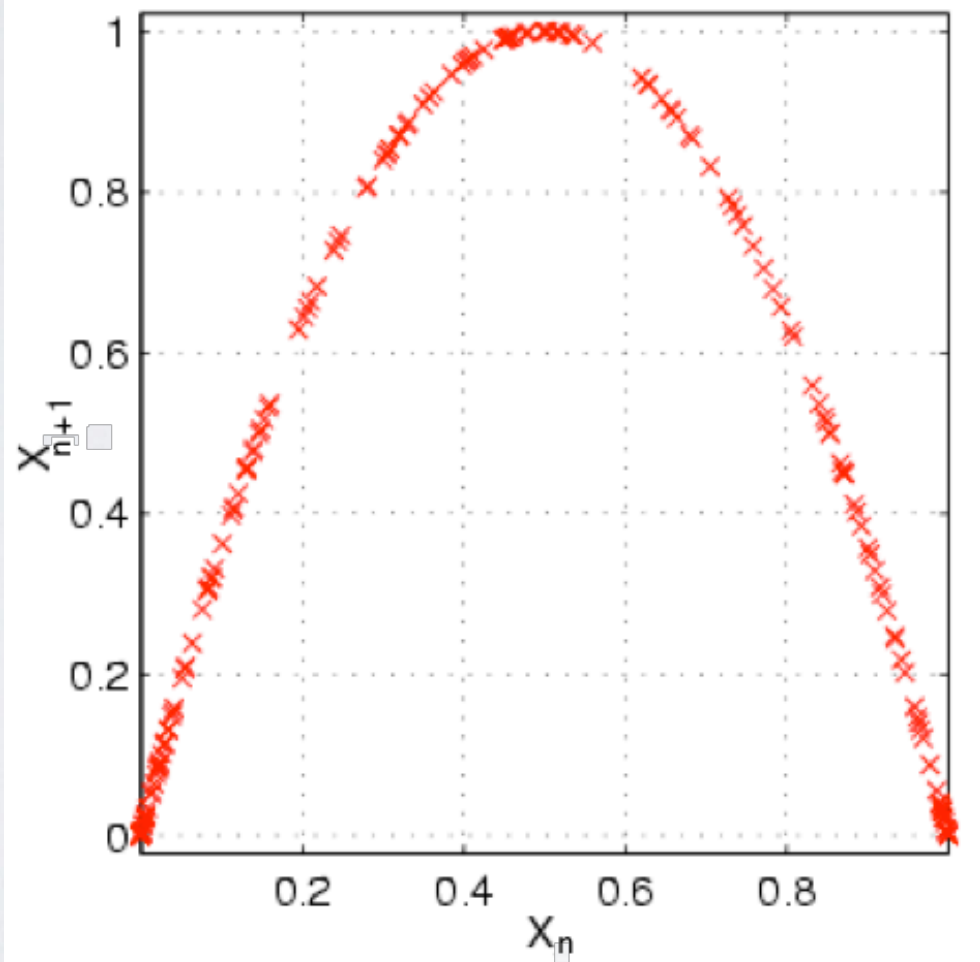

(b)

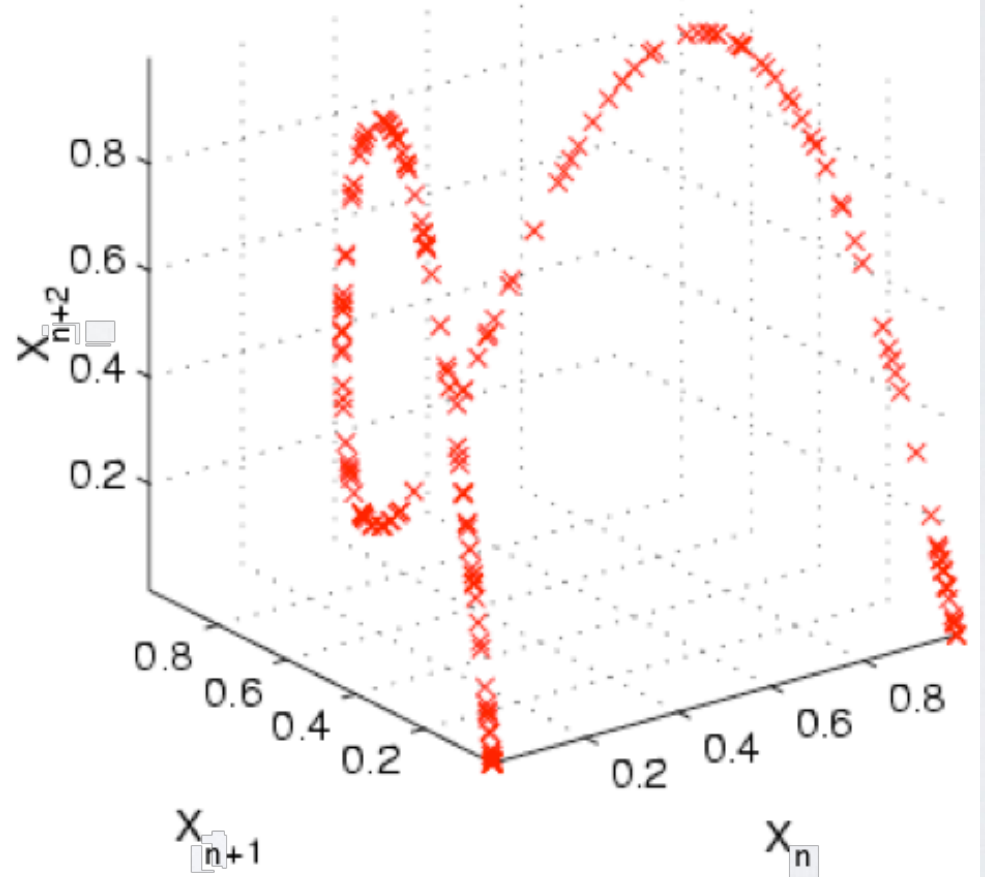




\section{IS THE BRAIN CHAOTIC? NEURINGER ANDVOSS}

- Graph of the Logistic Equation known as "Bifurcation Diagram".

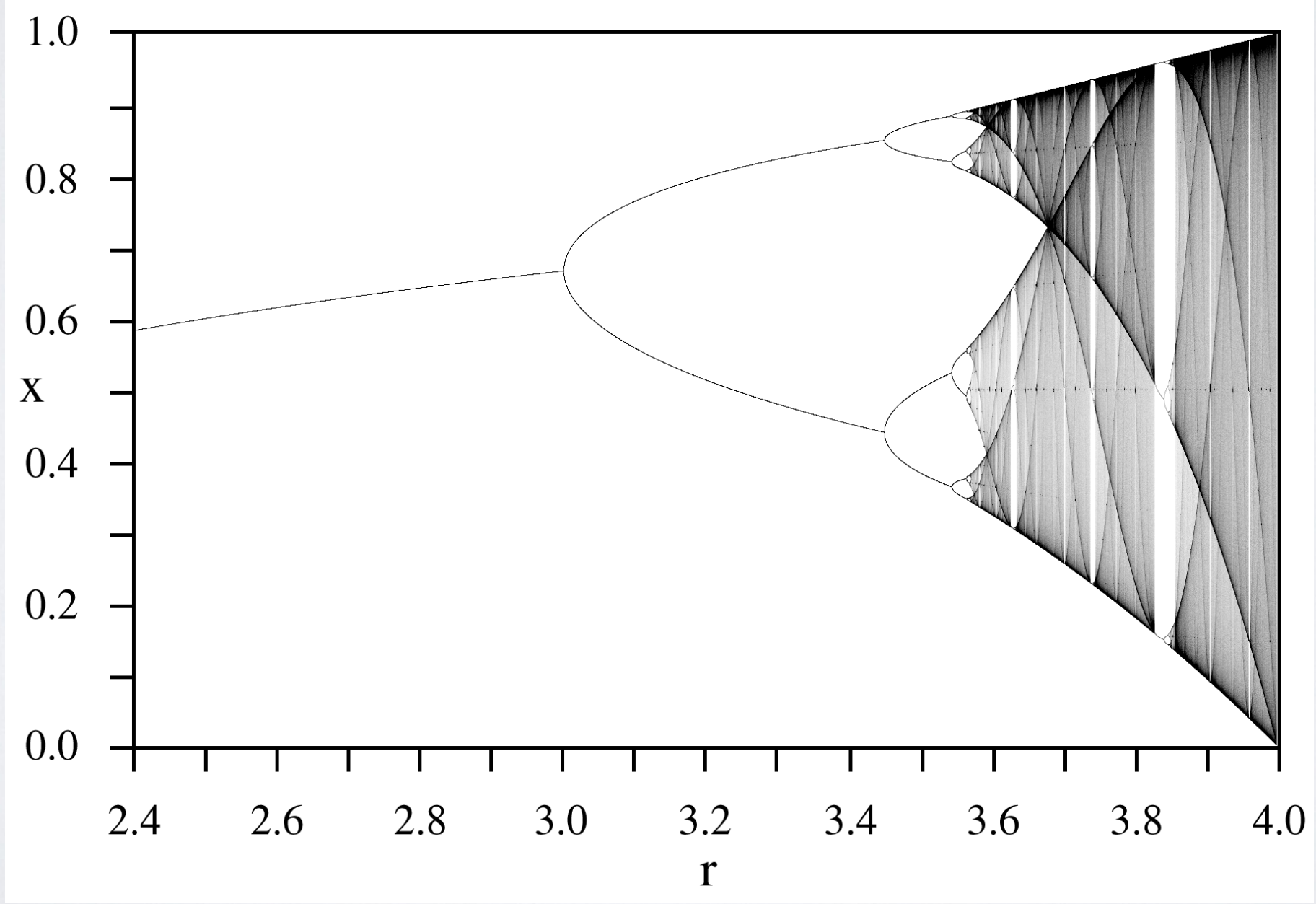




\section{IS THE BRAIN CHAOTIC? NEURINGER AND VOSS}

- Human behaviour does seem arbitrary and somewhat random; indeed, it is our apparent ability to arbitrarily choose this or that which leads us to believe that our choices are, pace compatibilists, in a sense "free".

- The beauty of postulating that humans are chaotic is that we can accommodate both points of view, that is, that humans are both spontaneous or random, and yet deterministic.

- Given that chaos mathematics aim to predict the behaviours of apparently unpredictable complex systems, it seems as if chaos mathematics are the right sort of explanation and predictive model for our behaviours. 


\section{CRITICISMS}

- How to quantify and plot peoples' choices? If I choose tea instead of coffee, or to write a philosophy paper instead of browsing Facebook, is that I.I or 1.2, or 3.5 , or 2.7 ?

- Neuringer et al.'s results were derived from a very small pool of experimental subjects.

- Two of the subjects, by Neuringer et al.'s own admission, never seemed to learn to produce chaotic sequences. 


\section{CRITICISMS}

- Metzger, the first author to respond to Neuringer et al. in the same journal, argues that Neuringer et al.'s experiment amounted to asking subjects to memorise the coordinates of a parabola's line. Ward and West (1994, p233) raise the same objection, and achieved the same results in a replication of Neuringer et al.'s experiment (Ward et al., 1994, pp233-235).

- The number of significant digits (decimal points) was insufficiently precise to generate a truly chaotic sequence. (WW 1994, p232). Recall: sensitivity to initial conditions and small changes... a decimal makes a difference in chaos. 


\section{CRITICISMS}

- Choosing numbers are not really canonical examples of free action; we tend to think of our choices as being morally pertinent, in cases where free-will actually matters.

And in such cases, it certainly seems that there would be many factors impinging on a moral agent, such that the single-variable Logistic Equation is hopelessly inadequate to cope with predicting which choice an agent would make. This objection, I think, is the most pertinent one. 


\section{YES, BUT ..}

- It seems to me that the problem with Neuringer et al. is not their idea (or indeed their conclusion), but rather, that it is their experimental methodology and setup that was problematic. Perhaps a more plausible demonstration would be to ask subjects to randomly choose numbers themselves without any prompting from a computer, and then see if the numbers chosen follow a chaotic equation's predictions. 


\section{AVALANCHES \\ INTHE BF \\ BRAIN}

66 The idea that the brain might be fundamentally disordered in some way first emerged in the late 1980s, when physicists working on chaos theory ... suggested it might help explain how the brain works.... Researchers built elaborate computational models to test the idea, but unfortunately they did not behave like real brains... In the 1990s, it emerged that the brain generates random noise, and hence cannot be described by deterministic chaos. When neuroscientists incorporated this randomness into their models, they found that it created systems on the border between order and disorder - self-organised criticality. More recently, experiments have confirmed that these models accurately describe what real brain tissue does. They build on the observation that when a single neuron fires, it can trigger its neighbours to fire too, causing a cascade or avalanche of activity that can propagate across small networks of brain cells. This results in alternating periods of quiescence and activity — remarkably like the build-up and collapse of a sand pile." (Robson, 2009, p2). 


\section{IN SHORT}

- We are chaos systems of a sort but our actions are precipitated, like an avalanche, rather than being completely random. And it is the precipitation of action, the ultimate final cause, which Libet calls "W', that is in need of explanation. And the answer seems to be chaos. 


\title{
THANK YOU!
}

\author{
John Ostrowick
}

\section{john@ostrowick.com}

\section{UCT}

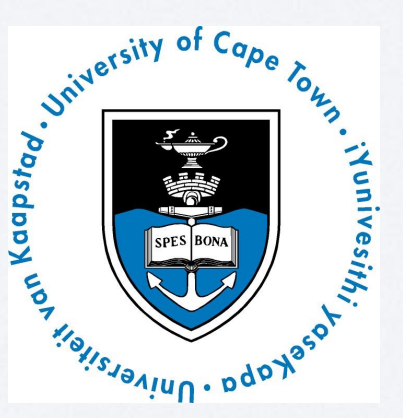

